\title{
Automated Structure Detection in HRTEM Images: An Example with Graphene
}

\author{
Jens Kling ${ }^{1}$, Jacob S. Vestergaard ${ }^{2}$, Anders L. Dahl², Thomas W. Hansen, Rasmus Larsen², \\ Jakob B. Wagner ${ }^{1}$ \\ ${ }^{1}$ Center for Electron Nanoscopy (DTU Cen), Technical University of Denmark, Kgs. Lyngby, Denmark \\ ${ }^{2}$ Image Analysis \& Computer Graphics, Department of Applied Mathematics and Computer Science \\ (DTU Compute), Technical University of Denmark, Kgs. Lyngby, Denmark
}

Graphene, as the forefather of 2D-materials, attracts much attention due to its extraordinary properties like transparency, flexibility and outstanding high conductivity, together with a thickness of only one atom. The properties seem to be dependent on the atomic structure of graphene and therefore characterizations on the atomic level are of interest. High-resolution transmission electron microscopy (HRTEM) is a state-of-the-art method to characterize the atomic structure of materials. Due to the inherently low mass-thickness of graphene, the contrast levels in the recorded images are often challenging to interpret. In order to increase the signal-to-noise ratio of the images two routes can be pursued: 1) the exposure time can be increased; or 2) acquiring series of images and summarize them after alignment. Both methods have the disadvantage of summing images acquired over a certain period of time making it difficult to resolve dynamic processes or unstable structures. Tools that assist to get the maximum of information out of recorded images are therefore greatly appreciated.

In order to get the most accurate results out of the structure detection, we have optimized the imaging conditions used for the FEI Titan ETEM with a monochromator and an objective-lens Cs-corrector. To reduce the knock-on damage of the carbon atoms in the graphene structure, the microscope was operated at $80 \mathrm{kV}$. As this strongly increases the influence of the chromatic aberration of the lenses, the energy spread of the electron gun was reduced. Using the monochromator an energy spread of $<0.2 \mathrm{eV}$ can be achieved. This gives a resolution better then $1.2 \AA$ which allow us to resolve the second order reflection of graphene and to visualize the atomic structure in HRTEM (fig. 1).

These images serve as a basis for the image analysis. Single-layer graphene with its regular honeycomb lattice is a perfect model structure to apply automated structure detection. By utilizing Fourier analysis the initial perfect hexagonal structure can easily be recognized. The recorded hexagonal tessellation reflects the unperturbed structure in the image. The centers of the C-hexagons are displayed as nodes. To segment the image into "pure" and "impure" regions, like areas with residual amorphous contamination or defects e.g. holes, a sliding window approach is used. The magnitude of the Fourier transformation within a window is compared to that of a perfect hexagonal tessellation. Areas where this relation exceeds a threshold are recognized as "impure" and a mask is created. As a result, the hexagonal tessellation overlays only the "pure" graphene structure in the image. 
As the real graphene structure is never perfect and undisturbed, at least at a length-scale of several $\mathrm{nm}$, the model structure has to be adjusted to the real structure. At this point, the image quality plays a crucial role. The algorithm assumes that irregularities in the graphene can be explained by a deformation in the xy-plane. To model this, a set of tensor B-splines is employed, which is deformed by matching model grid points with the C-hexagon centers. Dependent on the Cs and defocus-settings during microscopy these centers appear either dark or bright. One ends up with a deformed hexagonal tessellation, which can easily be transformed into a honeycomb lattice with the $\mathrm{C}$-atom positions included. As the microstructure is now available in the model, information like the C-C distance can be visualized as shown in fig. 2 .

Applying this method, the perfect graphene structure in recorded HRTEM-images can be determined fast and accurate over a wide length scale, and at the same time lattice deformations can be visualized. The method will be refined to facilitate the detection of larger defects like holes and the determination of the edge terminations.

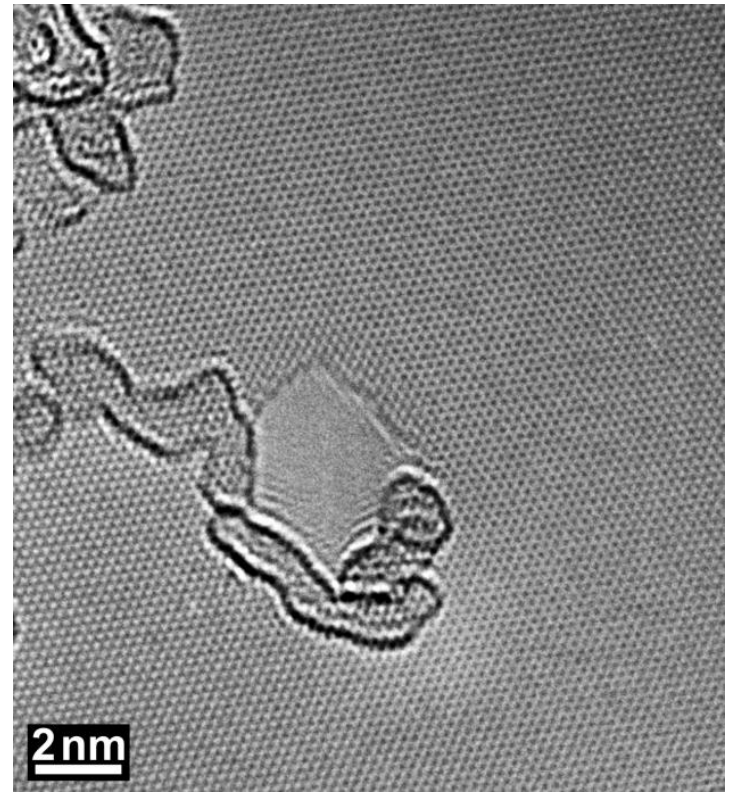

Figure 1. HRTEM image of graphene; Stack of 15 single images, background subtracted to reduce effect of inhomogeneous illumination, slightly Gauss-filtered.

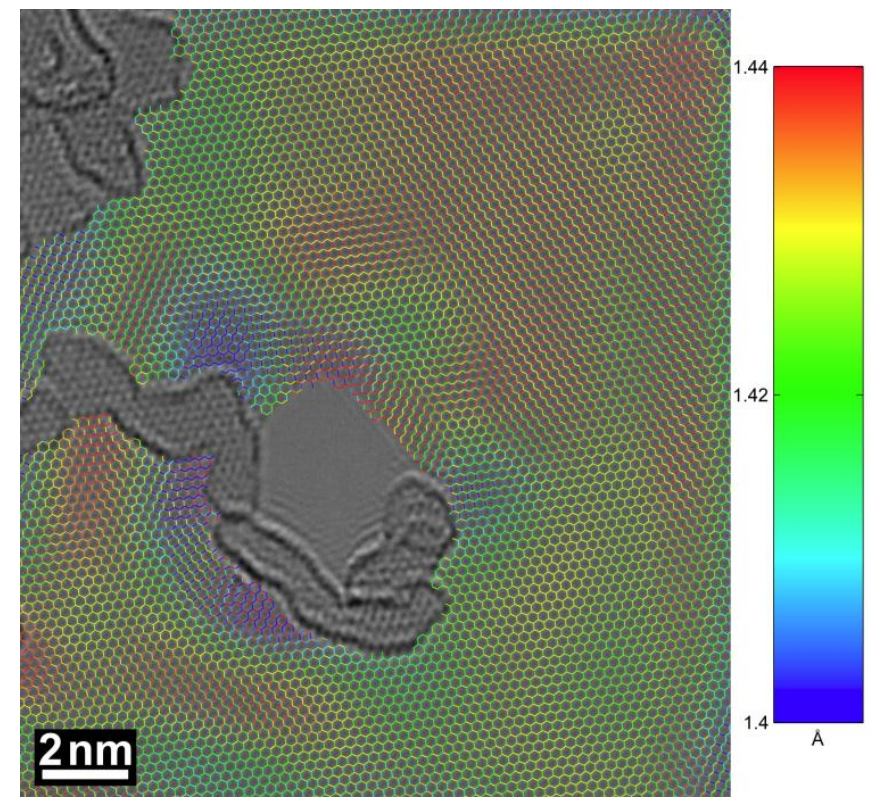

Figure 2. Image stack overlaid with hexagonal model structure from structure detection; the different colors visualize the difference in $\mathrm{C}-\mathrm{C}$ bond-distance. 